\section{- \\ NIST announces center to advance materials by design}

\author{
www.nist.gov/coe
}

$\mathbf{T}$ he National Institute of Standards and Technology (NIST) announced in December 2013 that it has selected a consortium led by Northwestern University to establish a new NISTsponsored center of excellence for advanced materials research. The new Center for Hierarchical Materials Design $(\mathrm{CHiMaD})$ will be funded in part by a $\$ 25$ million award from NIST over five years.

Other members of the CHiMaD consortium include the University of Chicago, the Northwestern-Argonne Institute of Science and Engineering (a partnership between Northwestern and the Department of Energy's Argonne National Laboratory), and the Computation Institute (a partnership between the University of Chicago and Argonne). The consortium also plans to work closely with QuesTek Innovations, a small business spin-off of Northwestern; ASM International; and Fayetteville State University.

"I'm particularly excited to announce this new alliance between NIST and two prominent research universities to drive innovation in the development of advanced materials," said Patrick Gallagher, Under Secretary of Commerce for Standards and Technology and NIST Director. "This new Center for Hierarchical Materials Design is a natural fit for NIST, which has a long tradition of serving as a nexus with academia and industry to advance research and innovation for the nation's benefit."

"The launch of this new center represents a major milestone in support of the President's Materials Genome Initiative and our national goal of doubling the pace of discovery and development of novel materials," said Cyrus Wadia, assistant director for Clean Energy and Materials R\&D at the White House Office of Science and Technology Policy. "By integrating the complementary strengths of computation, instrumentation, and creative modeling, this center promises to help keep America at the forefront of the materials revolution and a leader in the economically important domain of advanced manufacturing."

The new center will focus on developing the next generation of computational tools, databases, and experimental techniques to enable materials by design, one of the primary goals of the administration's Materials Genome Initiative (MGI). "Materials by design" employs physical theory, advanced computer models, vast materials properties databases, and complex computations to accelerate the design of a new material with specific properties for a particular application - perhaps an extremely tough, lightweight composite for auto bodies or a biocompatible cell scaffold for medicine. It stands in contrast to the traditional trial-and-error method of materials discovery.

Materials by design techniques have the potential to revolutionize the development of new advanced materials, which in turn have created whole industries. It is estimated that the average time from laboratory discovery of a new material to its first commercial use can take up to 20 years. The MGI aims to halve that.

The new center's work is expected to encompass both "hard" (inorganic) and "soft" (organic) advanced materials in fields as diverse as self-assembled biomaterials, smart materials for self-

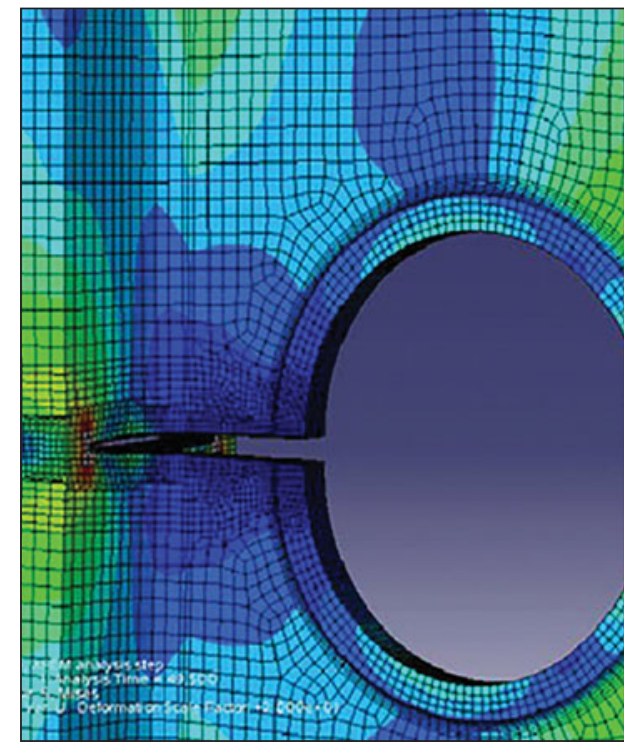

Computer model of crack growth in a part from a helicopter rotor. Colors indicate stresses surrounding the crack (red is high and blue is low). Such computations will be a key part of CHiMaD's research. Courtesy Northwesten University.

assembled circuit designs, organic photovoltaic materials, advanced ceramics, and metal alloys.

CHiMaD will focus these techniques on a particularly difficult challenge, the discovery of novel "hierarchical materials." Hierarchical materials exploit distinct structural details at various scales from the atomic on up to achieve special, enhanced properties. An example in nature of a hierarchical material is bone, a composite of mineral and protein at the molecular level assembled into microscopic fibrils that in turn are assembled into hollow fibers and on up to the highly complex material that is "bone."

The award to the Northwestern consortium for the Center for Hierarchical Materials Design is for $\$ 5$ million per year for five years, subject to available funds. NIST may, at its discretion, extend the award an additional five years after a performance review. The Northwestern-led consortium is contributing another approximately $\$ 4.65$ million to the center. 